Check for updates

Cite this: RSC Adv., 2018, 8, 31510

Received 15th July 2018

Accepted 26th August 2018

DOI: $10.1039 / \mathrm{c} 8 \mathrm{ra0} 0026 f$

rsc.li/rsc-advances

\section{Targeting HMGA protein inhibits retinoblastoma cell proliferation $\uparrow$}

\author{
Akilandeswari Balachandran, $t^{\mathrm{a}}$ Ajit Zambre, $t^{\mathrm{b}}$ Jagjot Singh Kainth, (D) be \\ Lakshmi Dhevi Nagarajha Selvan, ${ }^{c}$ Sowmya Parameswaran, ${ }^{d}$ Zahra Afrasiabi, \\ Subramanian Krishnakumar, ${ }^{\text {acd }}$ Raghuraman Kannan (D) *bf and Anandhi Upendran ${ }^{\text {tgh }}$
}

We describe a novel synthetic strategy for conjugating HMGA2 siRNA and the HMGA aptamer to the nucleolin aptamer and nucleolin antibody, respectively. Our studies demonstrate that these conjugates inhibit cell proliferation in retinoblastoma cells.
Retinoblastoma (RB) is a childhood eye cancer and approximately 9000 children are diagnosed every year. ${ }^{1}$ Current treatments include systemic chemotherapy using a combination of drugs such as vincristine, etoposide, and carboplatin with local chemotherapy of intravitreal melphalan and intra-arterial chemotherapy using carboplatin to save the vision of these children. These therapies result in severe toxicity to the retina and retinal pigment epithelial cells and in advanced stages, removal of the eye becomes inevitable. ${ }^{2-5}$ Therefore, it is important to identify targets that could complement the local chemotherapy to improve the efficiency, reduce the toxicity of these drugs and importantly prevent the vision loss. Studies have shown that targeted molecular therapy has great potential to improve the therapeutic outcome.$^{6}$ However, the limitation is a lack of a suitable vehicle for selective delivery of molecular therapeutics such as siRNA or aptamers that possess the ability to alter the functionality at genetic levels.

In retinoblastoma, the high mobility group A (HMGA) protein has been identified as an important therapeutic target, and is associated with invasiveness and metastasis of the

\footnotetext{
${ }^{a}$ Department of Nanobiotechnology, Vision Research Foundation, Kamalnayan Bajaj Institute for Research in Vision and Ophthalmology, Chennai, India. E-mail: drkk@ snmail.org; drkrishnakumar_2000@yahoo.com

${ }^{b}$ Department of Radiology, University of Missouri, Columbia, MO, USA. E-mail: kannanr@health.missouri.edu

${ }^{c}$ L\&T Ocular Pathology Department, Vision Research Foundation, Kamalnayan Bajaj Institute for Research in Vision and Ophthalmology, Chennai, India

${ }^{d}$ Radheshyam Kanoi Stem Cell Laboratory, Vision Research Foundation, Kamalnayan Bajaj Institute for Research in Vision and Ophthalmology, Chennai, India

${ }^{e}$ Department of Life Sciences, Lincoln University, Jefferson City, MO, USA

${ }^{f}$ Department of Biological Engineering, University of Missouri, Columbia, MO, USA

${ }^{g}$ Department of Medical Pharmacology and Physiology, University of Missouri, Columbia, MO, USA

${ }^{h}$ Institute of Clinical and Translational Sciences (MU-iCATS), School of Medicine, University of Missouri, Columbia, MO, USA. E-mail: upendrana@health.missouri.edu $\dagger$ Electronic supplementary information (ESI) available. See DOI: 10.1039/c8ra06026f

$\$$ Equal contribution to work.
}

disease. ${ }^{7}$ HMGA family proteins comprise two non-histone nuclear protein subsets, HMGA1 and HMGA2, and binds to AT-rich minor groove of the DNA. Upon binding, it alters the DNA chromatin structure and facilitates attachment of transcription factors leading to differential regulation of the transcription of genes. Previous studies indicated that RB tumors over express HMGA proteins and correlated with high risk histopathology features such as diffused invasion of the choroid and optic nerve. ${ }^{8,9}$ HMGA proteins are important target for molecular cancer therapy as these proteins are overexpressed only in tumors and not expressed in healthy adult tissues. ${ }^{10,11}$

HMGA proteins and mRNA transcripts can be selectively targeted using siRNA, aptamers, or DNA minor groove binders such as netropsin. ${ }^{12}$ Previous studies have shown that siRNA targeting HMGA2, promoted cell death through apoptosis and cell cycle arrest in cancer cells. ${ }^{13-15}$ Similarly, HMGA2 aptamer increased the sensitivity to gemcitabine treatment in pancreatic cancer cell lines. ${ }^{16}$ In RB tumours, HMGA aptamer reduced cell proliferation by activation of TGF $\beta$-SMAD4-mediated apoptotic pathway and a synergistic effect was also observed with etoposide. ${ }^{17}$ Indeed, our previous studies revealed that targeting HMGA at the transcript as well as protein levels compromised RB survival in in vitro. ${ }^{13,17}$ The selective delivery of either HMGA2 siRNA or HMGA aptamer to retinoblastoma can be achieved by targeting cell surface proteins those are overexpressed in RB cells. Previous studies have showed that nucleolin (NCL) is overexpressed on the surface of RB cells, and could be used for selective delivery of siRNA or aptamer. ${ }^{18}$ NCL shuttles from nucleolus to nucleoplasm, cytoplasm and cell surface. Targeting NCL needs a delivery vehicle and entry into the tumor cells.

Antibodies, peptides, or nanoparticles are commonly used as agents for delivery of siRNA or aptamer to cells. ${ }^{19-23}$ Antibodyaptamer pincers, antibody-siRNA conjugates, chemically programmable antibodies conjugated to RNA aptamers improve the affinity to target molecules. ${ }^{24-28}$ Aptamer conjugated with drugs are known; ${ }^{29-33}$ however, antibody-aptamer conjugates are sparse in the literature.$^{25}$ Although, the above 
listed strategies improved the delivery, ${ }^{34}$ the bottle-necks are: poor conjugation yield, and weak stability resulting in poor efficacy and increased toxicity. Therefore, it is important to overcome the above listed problems for successful delivery of siRNA or aptamer to RB tumor.

In this study, we synthesized and investigated two different delivery systems: NCL aptamer mediated delivery of HMGA2 siRNA (NCLap-HMGA2si) and NCL antibody mediated delivery of HMGA aptamer (NCLAb-HMGAap) for targeting HMGA in RB cells.

Synthesis of NCLap-HMGA2si and NCLAb-HMGAap are shown in Fig. 1. The newly synthesized conjugates were characterized; subsequently, stability, internalization, and in vitro toxicity were investigated. Internalization studies were performed in WERI-Rb-1 cells to validate the selective delivery of HMGA2si as well as HMGAap through NCLap and NCLAb respectively. Functional activity was demonstrated and compared with transfected HMGA2si or HMGAap in WERI-Rb-1 cells (described in ESI $\dagger$ ).

For synthesizing NCLap-HMGA2si conjugate, the $5^{\prime}$ end of NCL aptamer was modified with amine group using a spacer, and the $5^{\prime}$ end of HMGA2 siRNA was modified with thiol. ${ }^{35,36}$ The $3^{\prime}$ end of NCL aptamer was modified with fluorescein, while the cytosine and uracil bases of siRNA modified with 2 ' fluoro to improve its stability. For synthesizing NCLAb-HMGAap, we used amine modified HMGA2-cy5 aptamer and nucleolin antibody. ${ }^{37}$ The sequence of amine-NCL aptamer, thiol-HMGA2 siRNA, and amine modified HMGA aptamer is shown in ESITable $1 . \dagger$

As shown in Fig. 1, we synthesized NCLap-HMGA2si by treating equimolar concentrations of NCL aptamer and HMGA2 siRNA, in the presence of sulfo-SMPB bispecific linker. ${ }^{36}$ We analyzed the reaction mixture by gel electrophoresis. The conjugate showed gel retention at a higher molecular weight compared to that of free aptamer or siRNA. The presence of both NCL aptamer and HMGA2 siRNA in the conjugate were confirmed by respective bands in EtBr and fluorescein channels (Fig. 2A). The presence of bands corresponding to reactants suggests that the conjugation yield is only modest (40-50\%) and

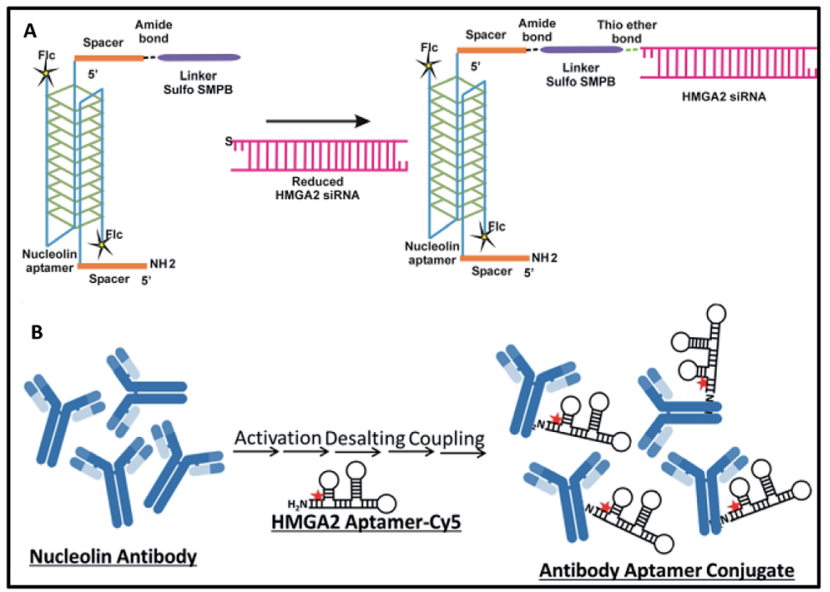

Fig. 1 Synthesis of (A) NCLap-HMGA2si; (B) NCLAb-HMGAap.

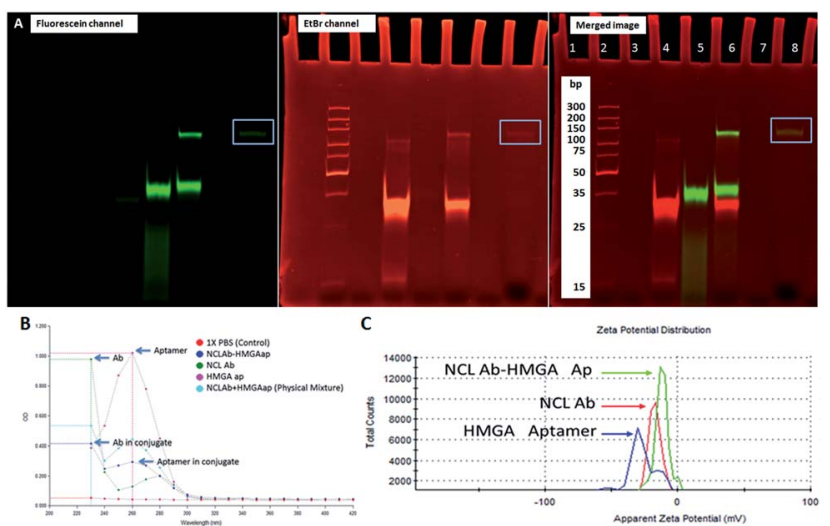

Fig. 2 Characterization of conjugates. (A) Non-denaturing PAGE of HMGA2 siRNA (lane 4), NCL aptamer (lane 5), unpurified NCLapHMGA2si (lane 6), and purified NCLap-HMGA2si (lane 8) showing the absence of free aptamer and siRNA in purified conjugate. (B) UV spectral and (C) zeta potential analysis of NCLAb-HMGAap, NCL antibody and HMGA aptamer exhibiting difference in conjugate compared to NCLab and HMGA aptamer.

required further purification. Previous literature showed that similar reactions, using sulfo-SMPB for conjugation of oligonucleotides with streptavidin, contain unreacted starting materials in the reaction mixture. ${ }^{38}$ To remove free aptamer and siRNA, NCLap-HMGA2si conjugate was excised from gel and purified by modified crush and soak method. ${ }^{39}$

The NCLAb-HMGAap conjugate was prepared using EDC/ sulfo-NHS procedure ${ }^{40}$ and the excess aptamer was removed by passing through centrifugal filter (30 kD Amicon). The NCLAb-HMGAap conjugate was characterized by gel electrophoresis, UV absorption, fluorescence and zeta potential measurements. The successful conjugation and isolation of pure NCLAb-HMGAap conjugate was confirmed by gel electrophoresis through fluorescent imaging (ESI-Fig. $1 \dagger$ ). The presence of individual components of the conjugate, NCLAb and HMGAap was confirmed by coomassie blue and gel red staining respectively (ESI-Fig. 2 and $3 \dagger$ ). The UV absorption spectrum of the conjugate showed characteristic peaks corresponding to antibody at $230 \mathrm{~nm}$ and aptamer at $260 \mathrm{~nm}$ (Fig. 2B). The zeta potential of the conjugate shifted towards less negative value $(-13 \pm 3 \mathrm{mV})$ compared to antibody $(-17 \pm 3 \mathrm{mV})$ and the aptamer $(-40 \pm 5 \mathrm{mV})$ confirming the formation of the conjugate. The zeta potential of NCLAb-HMGAap conjugate compared with free antibody and aptamer is shown in Fig. 2C. To confirm that conjugate contains both protein and aptamer, we quantified the protein using Bradford and Nanodrop, and aptamer by both fluorescent and Nanodrop measurements. The studies confirmed conjugation efficiency of $\sim 65 \%$ and $\sim 25 \%$ composition of the conjugate. There are fewer literature precedence on detailed characterization of the aptamer-oligo body conjugates $^{25}$ and therefore, the analytical measurements used to confirm NCLAb-HMGAap could serve as a predicate to analyze these types of conjugates.

We investigated the stability of both the conjugates, NCLapHMGA2si and NCLAb-HMGAap, in 1× PBS and 10\% FBS at $24 \mathrm{~h}, 48 \mathrm{~h}$, and $72 \mathrm{~h}$ and monitored using electrophoresis and 


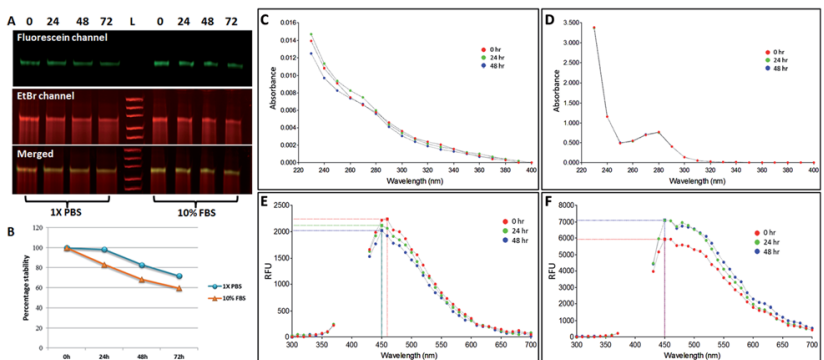

Fig. 3 Stability of the conjugates. (A) Non-denaturing PAGE demonstrate the stability of NCLap-HMGA2si conjugate in $1 \times$ PBS and $10 \%$ FBS at $0 \mathrm{~h}, 24 \mathrm{~h}, 48 \mathrm{~h}$ and $72 \mathrm{~h}$; (B) graphical presentation of the stability; (C) and (D) UV spectral analysis of NCLAb-HMGAap conjugates in $1 \times$ PBS and 10\% FBS at $0 \mathrm{~h}, 24 \mathrm{~h}$ and $48 \mathrm{~h}(\mathrm{E})$ and (F) Fluorescence spectra of NCLAb-HMGAap in $1 \times$ PBS and $10 \%$ FBS respectively.

optical spectroscopy (Fig. 3). The presence of single band in PAGE and stable peak in UV and fluorescence spectra confirmed the conjugates are stable.

The targeting ability of the newly synthesized conjugates were evaluated in WERI-Rb-1 cells by analyzing the receptor binding affinity. The cells were treated with the fluorescent labeled conjugates for two hours. Fluorescent microscopy was used to analyze the uptake of the conjugates in the cells. Colocalization of DAPI and FITC channels showed NCLapHMGA2si internalize in to the nuclear region (Fig. 4A). The surface and nuclear localization confirmed the binding ability of NCL aptamer to nucleolin protein present on the surface and subsequent internalization. Similar studies were performed with NCLAb-HMGAap conjugate to visualize aptamer internalization through NCL antibody. Co-localization of DAPI and CY-5 channels confirmed surface localization of the NCLAbHMGA2ap conjugate. Fluorescent images showed selective uptake of NCLAb-HMGAap conjugate on the surface of the WERI-Rb-1 cells confirming receptor mediated uptake of HMGA aptamer (Fig. 4B). The fluorescent image shows higher degree of cellular internalization of NCLAb-HMGAap conjugate compared to that of transfected HMGA aptamer. These results confirm that NCL antibody is effective in transporting the HMGA aptamer into the cells through the nucleolin receptors and retention of structural integrity.
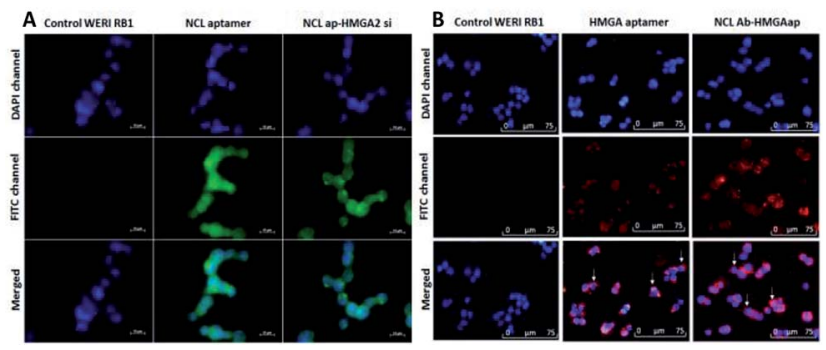

Fig. 4 Binding and internalization of conjugates. Fluorescence microscopic images of WERI-RB1 cells treated with (A) NCL aptamer and NCLap-HMGA2si showed surface as well as nuclear localization of both aptamer and conjugate; (B) cells transfected with HMGA aptamer and treatment of NCLAb-HMGAap conjugate revealed increased cell surface binding of the conjugate.
Table 1 In vitro cytotoxicity data showing $I_{50}(\mathrm{nM})$ when treated with WERI-Rb-1 cells

\begin{tabular}{lll}
\hline Conjugate & $\mathrm{IC}_{50}(\mathrm{nM})$ & $\mathrm{IC}_{50}\left(\mu \mathrm{gL}^{-1}\right)$ \\
\hline NCLap & 200 & 2.4 \\
HMGA2 siRNA & $>200$ & $>2.7$ \\
NCLap-HMGAsi & 72 & 1.0 \\
NCLAb & $>200$ & $>20$ \\
HMGAAp & 1400 & $>10$ \\
NCLab-HMGAap & 90 & 9.8 \\
PM (Ab + Ap) & $>200$ & $>20$
\end{tabular}

To investigate functional effects of the conjugates, in vitro cytotoxicity assays were performed in WERI-Rb-1 cells. MTT cell proliferation assay showed increased cytotoxicity in both the conjugates compared to HMGA2siRNA and HMGA aptamer (Table 1). NCLap-HMGA2si showed $\mathrm{IC}_{50}$ of $72 \mathrm{nM}(0.99 \mu \mathrm{g}$ $\left.\mathrm{ml}^{-1}\right)$, whereas, NCLAb-HMGAap showed $\mathrm{IC}_{50}$ of $90 \mathrm{nM}(9.75 \mu \mathrm{g}$ $\mathrm{ml}^{-1}$ ). Both NCLap-HMGA2si and NCLAb-HMGAap conjugates showed significant decrease in cell viability compared to their respective controls (Fig. 5A). The percentage cell viability for NCLap-HMGA2si conjugate was found to be $59 \%$ and $47 \%$ with $50 \mathrm{nM}$ and $100 \mathrm{nM}$ conjugate respectively. Likewise, the cell viability for NCLAb-HMGAap conjugate was $67 \%$ and $41 \%$ at $65 \mathrm{nM}$ and $130 \mathrm{nM}$ respectively (Fig. 5B). The enhanced cytotoxicity of the NCLap-HMGA2si conjugate is probably due to the synergistic effect of NCL aptamer and HMGA2 siRNA as both aptamer and siRNA are functional. ${ }^{13,18}$

We compared the cytotoxicity of NCLAb-HMGAap with free HMGA aptamer, NCL antibody and its physical mixture (Table 1). Based on the quantification data, we found that $90 \mathrm{nM}$ of NCLAb-HMGAap conjugate contains $26 \mathrm{nM}$ of HMGAap. The $\mathrm{IC}_{50}$ of NCLAb-HMGAap conjugate was $90 \mathrm{nM}$ suggesting that it is $\sim 50$ fold ( $p<0.001$; ESI-Fig. $4 \dagger)$ more cytotoxic compared to the free aptamer (1400 nM). The NCLAb showed no signs of cytotoxicity $\left(\mathrm{IC}_{50}>200 \mathrm{nM}\right)$; therefore, we believe that the enhanced cytotoxicity of the NCLAb-HMGAap conjugate could be attributed to targeted delivery of HMGA aptamer to RB cells. In addition to its role as a targeting vector, NCLAb camouflages the HMGA aptamer thereby facilitating the retention of structural integrity and functional property.
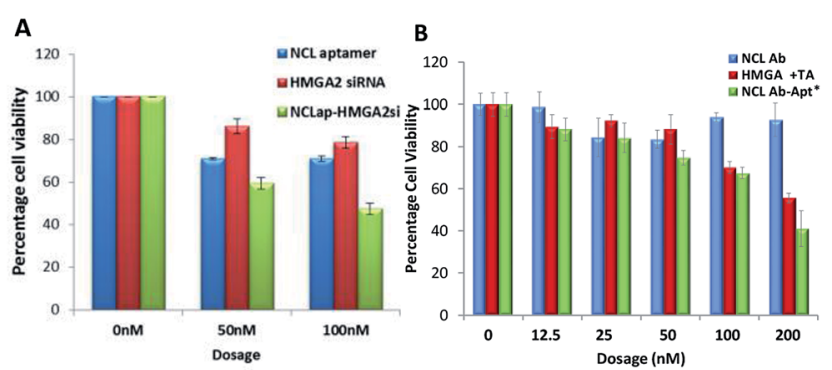

Fig. 5 Cytotoxicity of conjugates. (A) WERI-RB1 cells treated with NCLap-HMGAsi, NCL aptamer and HMGA2 siRNA. (B) WERI-RB1 cells treated with NCLAb-HMGAap, NCLAb, HMGA aptamer and physical mixture of NCLAb and HMGA aptamer. *Dosage represented here are based on pristine antibody concentration (ESI-Table $2 \uparrow$ ). 
In summary, we synthesized, characterized, and investigated the in vitro HMGA targeting ability of both NCL aptamer conjugated siRNA and NCL antibody conjugated aptamer in human RB cells. Our data demonstrate that the NCLAbHMGAap conjugate has unique and translatable characteristics compared to that of NCLAp-HMGA2si conjugate and listed as follows: (i) easier synthesis; (ii) scalable and better conjugation efficiency; (iii) higher degree of cellular internalization in WERIRB1 cells through receptor mediated internalization; and (iv) enhanced cytotoxicity, $\sim 50$ fold higher, in WERI-RB1 compared to free HMGA aptamer. Taken together, our data suggests that NCLAb-HMGAap is a potential candidate for future pre-clinical applications.

\section{Conflicts of interest}

There are no conflicts to declare.

\section{Acknowledgements}

This work is funded by research grant from Childhood Eye Cancer Trust (CHECT foundation), London, UK and Michael J and Sharon R Bukstein Cancer Research Endowment Funds.

\section{Notes and references}

1 https://wechope.org/retinoblastoma/retinoblastomaoverview/global-incidence/.

2 J. H. Francis, S. E. Brodie, B. Marr, E. C. Zabor, I. MondesireCrump and D. H. Abramson, Ophthalmology, 2017, 124, 488495.

3 H. A. Aziz, J. W. Kim, F. L. Munier and J. L. Berry, Ocul. Oncol. Pathol., 2017, 3, 34-40.

4 S. T. Michaels, T. A. Abruzzo, J. J. Augsburger, Z. M. Correa, A. Lane and J. I. Geller, J. Pediatr. Hematol. Oncol., 2016, 38, 65-69.

5 D. Susskind, U. Hagemann, M. Schrader, K. Januschowski, S. Schnichels and S. Aisenbrey, Acta. Ophthalmol., 2016, 94, 471-478.

6 A. Ferrario, M. Luna, N. Rucker, S. Wong, A. Lederman, J. Kim and C. Gomer, PLoS One, 2016, 11, e0153011.

7 P. Pallante, R. Sepe, F. Puca and A. Fusco, Front. Med. (Lausanne), 2015, 2, 15.

8 G. Mu, H. Liu, F. Zhou, X. Xu, H. Jiang, Y. Wang and Y. Qu, Hum. Pathol., 2010, 41, 493-502.

9 N. Venkatesan, M. Kandalam, G. Pasricha, V. Sumantran, G. Manfioletti, S. J. Ono, M. A. Reddy and S. Krishnakumar, J. Pediatr. Hematol. Oncol., 2009, 31, 209214.

10 A. Fusco and M. Fedele, Nat. Rev. Cancer, 2007, 7, 899-910.

11 R. Sgarra, A. Rustighi, M. A. Tessari, J. Di Bernardo, S. Altamura, A. Fusco, G. Manfioletti and V. Giancotti, FEBS Lett., 2004, 574, 1-8.

12 Y. Miao, T. Cui, F. Leng and W. D. Wilson, Anal. Biochem., 2008, 374, 7-15.

13 N. Venkatesan, S. Krishnakumar, P. Deepa, M. Deepa, V. Khetan and A. M. Reddy, Mol. Vision, 2012, 18, 2420-2437.
14 S. Esmailzadeh, B. Mansoori, A. Mohammadi, D. Shanehbandi and B. Baradaran, J. Gastrointest. Cancer, 2017, 48, 156-163.

15 Z. Shi, D. Wu, R. Tang, X. Li, R. Chen, S. Xue, C. Zhang and X. Sun, J. Biosci., 2016, 41, 229-236.

16 M. Watanabe, S. Sheriff, K. B. Lewis, S. L. Tinch, J. Cho, A. Balasubramaniam and M. A. Kennedy, Cancer Lett., 2012, 315, 18-27.

17 V. Nalini, P. R. Deepa, R. Raguraman, V. Khetan, M. A. Reddy and S. Krishnakumar, Ocul. Oncol. Pathol., 2016, 2, 262-269.

18 N. Subramanian, A. Srimany, J. R. Kanwar, R. K. Kanwar, B. Akilandeswari, P. Rishi, V. Khetan, M. Vasudevan, T. Pradeep and S. Krishnakumar, Mol. Ther.-Nucleic Acids, 2016, 5, e358.

19 J. Zhou and J. Rossi, Nat. Rev. Drug Discovery, 2017, 16, 181202.

20 K. Chen, B. Liu, B. Yu, W. Zhong, Y. Lu, J. Zhang, J. Liao, J. Liu, Y. Pu, L. Qiu, L. Zhang, H. Liu and W. Tan, Wiley Interdiscip. Rev.: Nanomed. Nanobiotechnol., 2017, 9, e1438.

21 L. Yang, X. Zhang, M. Ye, J. Jiang, R. Yang, T. Fu, Y. Chen, K. Wang, C. Liu and W. Tan, Adv. Drug Delivery Rev., 2011, 63, 1361-1370.

22 J. G. Bruno, Pharmaceuticals, 2013, 6, 340-357.

23 M. P. Melancon, M. Zhou, R. Zhang, C. Xiong, P. Allen, X. Wen, Q. Huang, M. Wallace, J. N. Myers, R. J. Stafford, D. Liang, A. D. Ellington and C. Li, ACS Nano, 2014, 8, 4530-4538.

24 S. Kang and S. S. Hah, Bioconjugate Chem., 2014, 25, 14211427.

25 K. Heo, S. W. Min, H. J. Sung, H. G. Kim, H. J. Kim, Y. H. Kim, B. K. Choi, S. Han, S. Chung, E. S. Lee, J. Chung and I. H. Kim, J. Controlled Release, 2016, 229, 1-9.

26 U. Wuellner, J. I. Gavrilyuk and C. F. Barbas 3rd, Angew. Chem. Int. Ed. Engl., 2010, 49, 5934-5937.

27 Y. Wan, L. Wang, C. Zhu, Q. Zheng, G. Wang, J. Tong, Y. Fang, Y. Xia, G. Cheng, X. He and S. Y. Zheng, Cancer Res., 2018, 78, 798-808.

28 S. Dickgiesser, N. Rasche, D. Nasu, S. Middel, S. Horner, O. Avrutina, U. Diederichsen and H. Kolmar, ACS Chem. Biol., 2015, 10, 2158-2165.

29 K. Chen, B. Liu, B. Yu, W. Zhong, Y. Lu, J. Zhang, J. Liao, J. Liu, Y. Pu, L. Qiu, L. Zhang, H. Liu and W. Tan, Wiley Interdiscip. Rev.: Nanomed. Nanobiotechnol., 2017, 9(3), DOI: 10.1002/wnan.1438.

30 R. Wang, G. Zhu, L. Mei, Y. Xie, H. Ma, M. Ye, F. L. Qing and W. Tan, J. Am. Chem. Soc., 2014, 136, 2731-2734.

31 S. Yoon, K. W. Huang, V. Reebye, D. Spalding, T. M. Przytycka, Y. Wang, P. Swiderski, L. Li, B. Armstrong, I. Reccia, D. Zacharoulis, K. Dimas, T. Kusano, J. Shively, N. Habib and J. J. Rossi, Mol. Ther.-Nucleic Acids, 2017, 6, $80-88$.

32 N. Zhao, S. N. Pei, J. Qi, Z. Zeng, S. P. Iyer, P. Lin, C. H. Tung and Y. Zu, Biomaterials, 2015, 67, 42-51.

33 G. Zhu, G. Niu and X. Chen, Bioconjugate Chem., 2015, 26, 2186-2197.

34 J. H. Jeong, H. Mok, Y. K. Oh and T. G. Park, Bioconjugate Chem., 2009, 20, 5-14. 
35 C. L. Esposito, S. Catuogno and V. de Franciscis, Journal of RNAi and Gene Silencing: An International Journal of RNA and Gene Targeting Research, 2014, 10, 500-506.

36 W. Y. Lai, W. Y. Wang, Y. C. Chang, C. J. Chang, P. C. Yang and K. Peck, Biomaterials, 2014, 35, 2905-2914.

37 M. Brinkley, Bioconjugate Chem., 1992, 3, 2-13.
38 C. M. Niemeyer, T. Sano, C. L. Smith and C. R. Cantor, Nucleic Acids Res., 1994, 22, 5530-5539.

39 Z. Chen and D. E. Ruffner, Biotechniques, 1996, 21, 820-822. 40 G. T. Hermanson, in Bioconjugate Techniques, Academic Press, Boston, 3rd edn, 2013, pp. 549-587. 